\title{
HUMAN VISION THRESHOLDING WITH ENHANCEMENT FOR DARK BLURRED IMAGES FOR LOCAL CONTENT PRESERVATION
}

\author{
Chelsy Sapna Josephus and Remya .S \\ Department of Computer Science, University of Kerala, Thiruvananthapuram, \\ India \\ chelze4u@gmail.com, remyayes@gmail.com
}

\begin{abstract}
There are several images that do not have uniform brightness which pose a challenging problem for image enhancement systems. As histogram equalization has been successfully used to correct for uniform brightness problems, a histogram equalization method that utilizes human visual system based thresholding(human vision thresholding) as well as logarithmic processing techniques were introduced later. But these methods are not good for preserving the local content of the image which is a major factor for various images like medical images.Therefore new method is proposed here. This method is referred as "Human vision thresholding with enhancement technique for dark blurred images for local content preservation”. It uses human vision thresholding together with an existing enhancement method for dark blurred images. Experimental results shows that the proposed method outperforms the former existing methods in preserving the local content for standard images and medical images
\end{abstract}

\section{KEYWORDS}

Adaptive histogram equalization, human visual system, human vision thresholdin ,enhancement ,local content preservation.

\section{INTRODUCTION}

Image enhancement is necessary to improve the visual appearance of the image .It also gives a good transform representation for automated image processing such as analysis and segmentation of images [1-2]. To discern the concealed but important information in the images, it is needed that the usage of various image enhancement methods such as enhancing edges, emphasizing the differences, or reducing the noise is done. Processing techniques for image enhancement can be classified into spatial domain enhancement and transform domain enhancement methods. Adaptive histogram equalization(AHE) [3] belongs to spatially non uniform enhancement technique. In Spatial domain enhancement methods, the direct manipulation of image intensity values is done. While the spatially uniform methods use a transformation applied to all pixels of the image, the later methods use an input output transformation that varies adaptively with the local characteristics of the image. Histogram equalization has a disadvantage that it is not suitable for retaining local detail of an image. Histogram equalization tends to over-enhance the image contrast if there is a high peaks in the histogram resulting in a undesired loss of visual data, of quality and of intensity scale. Also minute details related to small bins of the histogram are

Natarajan Meghanathan, et al. (Eds): ITCS, SIP, JSE-2012, CS \& IT 04, pp. 251-262, 2012.

(C) CS \& IT-CSCP 2012 
eliminated. AHE applies locally varying gray-scale transformation on each small region of the image. This method does not completely eliminate noise enhancement in smooth regions.

Local histogram equalization (LHE) is one of the popular branches for local image enhancement. Generally, LHE uses a small window to define a contextual region (CR) for the centre pixel of that window .Only the block of pixels that fall in this window is taken into the account for the calculation of cumulative density function(CDF). Therefore, as the window slides, the CDF is modified. The CDF is the main contributor for the LHE transform function. Hence, in LHE, the transform function of a pixel is depending on the statistics of its neighbors in CR. Because the transform function changes as a response to the changes in the contents of CR, LHE is also popularly known as adaptive histogram equalization (AHE)

Multi histogram Equalization is another efficient way of enhancing an image. But Multihistogram equalization has generally been limited to bi-histogram equalization, and it has been previously proved that tri-histogram equalization does not have any consistent advantage. So in the previous methods the introduction of the human visual system based multi-histogram equalization method is done. This algorithm focusses on the advantages of multi-histogram equalization with the benefit of an effective quantitative measure to ensure optimal results while removing useless information to avoid the production of unwanted artifacts. This method overcomes this limitation by separating the image into different regions of illumination instead of thresholding by simple pixel intensity values. In this manner, histogram equalization can be done on each region to correct for non uniform illumination. In order to perform this segmentation, model of the human visual system is utilized. HVS-based image enhancement aims to specify the way in which the HVS discriminates between useful and unwanted data.

\section{METHODS}

\subsection{HUMAN VISUAL SYSTEM BASED IMAGE ENHANCEMENT(HVS)[13]}

Human Visual System (HVS) based Image Enhancement aims to emulate the way in which the human visual system diffferentiates between useful and unwanted data and it is based on the background illumination and the gradient of an image. The former is arrived at using the following formula:

$$
\mathrm{B}(\mathrm{x}, \mathrm{y})=\left[\frac{1}{2}\left(\frac{1}{4} \sum_{Q} X(i, j)+\frac{1}{4 \sqrt{2}} \sum_{Q} X(k, l)\right)+X(x, y)\right]+2
$$

where $\mathrm{B}(\mathrm{x}, \mathrm{y})$ is the background intensity at each pixel, $\mathrm{X}(\mathrm{x}, \mathrm{y})$ is the input image, $\mathrm{Q}$ is all of the pixels which are directly up, down, left, and right from the pixel, and $\mathrm{Q}^{\prime}$ is all of the pixels diagonally one pixel away. Here a parameter BT, which is the difference in the maximum and minimum graylevels of an image is also defined and is arrived at using:

$\mathrm{BT}=\max (\mathrm{X}(\mathrm{x}, \mathrm{y}))-\min (\mathrm{X}(\mathrm{x}, \mathrm{y}))$

Further, the gradient information is also needed, which is arrived at in the following formula:

$\mathrm{G} 1=\mathrm{X}(\mathrm{x}, \mathrm{y})-\mathrm{X}(\mathrm{x}, \mathrm{y}+1)$

$\mathrm{G} 2=\mathrm{X}(\mathrm{x}, \mathrm{y}) \mathrm{X}(\mathrm{x}+1, \mathrm{y})$ 
$\mathrm{X}^{\prime}(\mathrm{x}, \mathrm{y})=(|\mathrm{G} 1|+|\mathrm{G} 2|) / 2$

where $\mathrm{X}^{\prime}(\mathrm{x}, \mathrm{y})$ is the gradient information and G1, G2 are the directional gradients. Finally, we must also take into consideration some parameters concerning the human eye itself, which is referred as $\mathrm{Bxi}, \mathrm{i}=1,2,3$ and $\mathrm{Ki}, \mathrm{i}=1,2,3$. These are arrived at using the following formulas:

$$
\begin{aligned}
& B_{x 1}=\alpha_{1} B_{T} \\
& B_{x 2}=\alpha_{2} B_{T} \\
& B_{x 3}=\alpha_{3} B_{T} \\
& K 1=\frac{1}{100} * \beta * \max \left(\frac{X^{\prime}(x, y)}{B(x, y)}\right) \\
& K 2=K 1 \sqrt{B_{x 2}} \\
& K 3=\frac{K 1}{B_{x 3}}
\end{aligned}
$$

Where $\alpha_{1}, \alpha_{2}, \alpha_{3}$ are parameters based upon the three distinct regions of response characteristics displayed by the human eye. As a, is the lower saturation level, it is effective to set this to 0 . Here $\alpha_{2}, \alpha_{3}$, is determined experimentally.

Using this information, the image is first broken up into the different regions of human visual response. These different regions are characterized by the minimum difference between two pixel intensities for the human visual system to register a difference. The next step is to threshold the three regions, removing the pixels which do not constitute a noticeable change for a human observer and placing these in a fourth image. These four images are arrived at using the following formula:

$\operatorname{Im} 1=\mathrm{X}(\mathrm{x}, \mathrm{y})$ such that $B x 2 \geq B(x, y) \geq B x 1 \& \frac{X^{\prime}(x, y)}{\sqrt{B(x, y)}} \geq K 2 \ldots(12)$
$\operatorname{Im} 2=\mathrm{X}(\mathrm{x}, \mathrm{y})$ such that $B x 3 \geq B(x, y) \geq B x 2 \& \frac{X^{\prime}(x, y)}{B(x, y)} \geq K 1 \ldots .(13)$
$\operatorname{Im} 3=\mathrm{X}(\mathrm{x}, \mathrm{y})$ such that $B(x, y) \geq B x 3 \& \frac{X^{\prime}(x, y)}{(B(x, y))^{2}} \geq K 3 \ldots \ldots \ldots(14)$

$\operatorname{Im} 4=X(x, y) \quad$ All Remaining pixels 
These four images are then enhanced separately and recombined to form the enhanced image. The above mentioned thresholding method is also referred as human vision thresholding .

HVS based histogram Equalization is a method that uses the concept of multi-histogram equalization. By separating the image into regions by the quality of illumination, such as overilluminated, well illuminated, and under-illuminated, traditional histogram equalization can be used on each region to correct for non-uniform illumination.For this, the utilization of the human visual system to segment the image, using the measure of image enhancement to select $\alpha_{2}$ and $\alpha_{3}$ is done. The first three images are then equalized separately and unionized, with the remaining pixels filled in. In summary, the algorithm is executed as follows:

1. Obtain the input image and segment image using Human Vision Thresholding algorithm

2. Equalize the three images separately.

3. Recombine the pixels in the three equalized images

4. Fill in the missing pixels to generate the output image .

\subsection{HUMAN VISION BASED SEGMENTATION WITH EDGE PRESERVING CONTRAST ENHANCEMENT(EPCE) (HVSedge)[12]}

This is another method which uses the human vision based thresholding for segmentation. This method is a variation of HVS method. Here unlike the HVS method, the images $\operatorname{Im} 1, \operatorname{Im} 2$ and $\operatorname{Im} 4$ satisfying the corresponding thresholding conditions as in HVS method is considered. Then one of the images obtained is enhanced by edge preserving enhancement method and the remaining images are enhanced using classical histogram equalization method .

$\operatorname{EPCE}$ (Edge preserving contrast enhancement) is a enhancement algorithm which is designed to preserve edges while improving contrast locally by combining the output of an edge-detection algorithm with the original spatial information of the image. This achieves a more robust enhancement algorithm that is able to perform edge detection or enhancement. This enhancement algorithm can be used with any suitable edge-detection algorithm. It uses preprocessing steps to standardize image brightness and several post processing steps to enhance the edges contained.

\section{Step1 :}

Initially the following operation is done on each image pixel, using the following formula (eqn 15).This operation is based on the local mean at each image pixel.

$I(x, y)=\frac{2}{1+e^{2 \tau(x, y) / \lambda(x, y)}}-1$

where $\mathrm{I}(\mathrm{x}, \mathrm{y})$ is the output image, $\tau(\mathrm{x}, \mathrm{y})$ is the gray scale image, and $\lambda$ is the local statistic of the image used to adjust the transfer function to the local mean. Finally, $\lambda$ is

$\lambda(x, y)=C+(M-C)\left(\frac{\mu(x, y)}{M}\right)$

where $\mathrm{C}$ is a user-selected enhancement parameter, with effective range $0=\mathrm{C}<256, \mathrm{M}$ is the maximum value of the range, and $\mu(\mathrm{x}, \mathrm{y})$ is the local mean of the image. 


\section{Step 2 :}

To enhance the contrast a high-pass filter is applied on the image. This image is called $\mathrm{I}_{\mathrm{EN}}$. Similarly an application of edge detection algorithm is applied to the another copy of the image obtained from step1, resulting in a second image which is referred as $\mathrm{I}_{\mathrm{ED}}$.

\section{Step 3:}

Finally, the following formula gives the output-enhanced image:

$I_{\mathrm{FEN}}=\mathrm{A}\left(\mathrm{I}(\mathrm{x}, \mathrm{y})+\mathrm{I}_{\mathrm{ED}}(\mathrm{x}, \mathrm{y})^{\gamma} \times \mathrm{I}_{\mathrm{EN}}(\mathrm{x}, \mathrm{y})^{\alpha}\right)$

Where $\mathrm{I}_{\text {FEN }}$ is the output image and $\mathrm{A}, \alpha$, and $\gamma$ are user defined operating parameters. Selection of $\mathrm{M}$ and $\mathrm{C}$ can be done simply and quickly by a human; however, selecting $\alpha$ and $\gamma$ is a more time-consuming task.(, $\mathrm{M}=260$ and $\mathrm{C}=50)$. This method preserves the local content of the input image in the output image better than HVS method .

Since the local content preservation is of utmost concern in the case of medical images, new methods for the same need to introduced .In the next section, an extension of this HVS method referred as HVSEDBI is proposed, which preserves the local content better than the former above mentioned methods. The proposed HVSEDBI method is a variation of the classical HVS method . Even though the classical HVS method is a good method for enhancement, it does not preserve the local content of an image . Further more an another extension of this method called HVSedge ,though preserves the content better than classical HVS method, does not preserve the contents much in the case of images like medical images where local content preservation is of utmost importance. Therefore HVSEDBI is introduced here. This method uses the enhancement scheme for dark blurred images along with the edge preserving contrast enhancement method. The proposed method is applied to different category of grey scale images like medical images and other standard images .

\section{PROPOSED METHOD}

\subsection{HUMAN VISION THRESHOLDING WITH ENHANCEMENT FOR DARK BLURRED IMAGES FOR LOCAL CONTENT PRESERVATION (HVSEDBI)}

Another extension of the HVS method is proposed here. In this method the segmentation of the original image into 3 images is done by means of human visual system based(or human vision ) thresholding. In addition to this after the three images are obtained using the thresholding (Im1 ,Im2 and Im4), the second image is enhanced by using normal histogram equalization, the third image using the edge preserving contrast enhancement method( mentioned in the previous section) and the third image is enhanced by using the following enhancement scheme .

In this enhancement scheme there are mainly 2 steps applied to the first image. The first step is to apply unsharp masking to the original image to get a sharper and more detailed image. Second step is contrast enhancement step that is based on a mapping function. In the following section the description of these steps are given in detail.

The steps of this enhancement technique is as follows: 


\section{Step 1:}

Unsharp masking step: This step enhances the small structures and bring out the hidden details in the image by using unsharp masking. It only sharpens the areas, which have edges or large amount of details. In unsharp masking a blurred copy of the original image is generated by using laplacian filter and then subtracted from the original image. This is given by the following formula .

$I(i, j)=I_{O}(i, j)-I_{b}(i, j)$

where $I(i, j)$ is the unsharp masking image, $I_{O}(i, j)$ is the original image,and $I_{b}(i, j)$ is the blurred copy.

Here before passing to the contrast enhancement step the unsharp masked image is multiplied by a value, and added to the original image to get the image that will be contrasted. Here, the large features are not altered much, but the smaller ones are enhanced. The result is a sharperand more detailed image.This operation is given by the following formula .

$$
g(i, j)=I(i . j)+k I_{O}(i, j) \text {. }
$$

where $g(i, j)$ is output image, $k$ is the scaling constant. ' $k$ 'takes up values between 0.2 and 0.7

\section{Step 2:}

Contrast enhancement step: In this step , a sliding map window( $3 \times 3$ window) is moved from the left side to the right side of original image horizontally. A pixel value in the enhanced widow depends only on its value. This means that if the pixel of interest exceeds a certain threshold value, its value remain unchanged but if the value of the pixel is under the threshold then it will be remapped to a new value. The process can be described with the mapping function $\mathrm{M}$ defined below.

$$
\mathrm{O}=\mathrm{M}(\mathrm{i})
$$

where $\mathrm{O}$ and $\mathrm{i}$ are the new and old pixel values, respectively. The mapping function $\mathrm{M}$ is given by the following equation

$$
\mathrm{M}=\mathrm{i}^{*} \mathrm{c} / 1+\mathrm{e}^{-\mathrm{i}}
$$

According to above mapping function the new value of corresponding pixel will be:

$$
O= \begin{cases}i & \text { if } \mathrm{i}>\mathrm{t} \\ i+\left(i *\left(\frac{c}{1+e^{-i}}\right)\right. & \text { if } \mathrm{i}<\mathrm{t}\end{cases}
$$

where $\mathrm{c}$ is a contrast factor which determines the degree of the contrast that is needed . After map window reaches the right side of the image, it returns to the left side and moves down a step below. The process is repeated until the sliding window reaches the right-bottom corner of the entire image. On careful observation of the results, it is seen that local content preservation by 
this method is far better than the HVS and HVSedge method. The enhancement of contrast by this method is also comparatively better.

\section{RESULTS AND DISCUSSIONS}

For the experiment several categories of medical images are being used .Here an analysis based on brain images, mammograms and a knee images are given .In addition to this the result of application of this method to the well known images like $600 \times 600$ pixel Einstein girl image and other images are also specified .For EPCE used for enhancing one of the thresholded images, A is taken as 40,gamma=1,alpha $=1, \mathrm{C}=240, \mathrm{M}=255$.Generally for HVS thresholding $\alpha_{2}=0.1, \alpha_{1}=0$, $\alpha_{3}=0$,beta $=-1.5$.

The performance measures used here are Peak Signal to Noise Ratio (PSNR)and Average mean brightness error (AMBE) and Contrast improvement index(CII). The measure AMBE is mainly taken because it is used to specify the local content preservation . So if AMBE value obtained by a particular method is lesser compared to other methods, then the former method preserves the local content of the input image in the output image better. Generally the PSNR value of a image $R$ with respect to the original image $\mathrm{O}$, both of size $\mathrm{M} \times \mathrm{N}$, is calculated as shown below.

$$
\begin{array}{r}
\text { MSE = } \sum_{m, n}[O(m, n)-R(m, n)]^{2} /(M * N) \\
P S N R=10 * \log 10\left(255^{2} / M S E\right)
\end{array}
$$

Where MSE is the mean squared error given by

The Average mean brightness error (AMBE) is basically used to measure the capability of the method to maintain the mean brightness of the input image in its output image.

The AMBE is given by: $\mathrm{AMBE}=\left|\mathrm{X}^{\prime}-\mathrm{Y}^{\prime}\right|$

where $X^{\prime}$ and $Y^{\prime}$ are the average intensity values of the input and output image, respectively. The brightness of the input image should be retained in the output image in order to avoid intensity saturation problem. Hence, a good enhancement method should have a low value of AMBE.

Contrast improvement index (CII) is used to measure the increase in the contrast generated by the methods .For this a $3 \mathrm{X} 3$ window is taken and passed over the entire image. At each window the maximum and minimum intensity value is found. The summation of all the maximum values and the summation of all the minimum values is obtained for the entire image. Then the ratio of (maximum-minimum) and (maximum+minimum) is taken and multiplied by $1 /$ number of windows. Finally the ratio of this above obtained value for the input and output image gives the CII value for the image obtained by the corresponding method . 
Table 1 Comparison of the AMBE values produced by (HVS) and HVSedge and the proposed method HVSEDBI

\begin{tabular}{|l|l|l|l|}
\hline \multirow{2}{*}{ Image } & \multicolumn{3}{|c|}{ AMBE } \\
\cline { 2 - 4 } & HVS & HVSedge & HVSEDBI \\
\hline Lena & 34.75 & 26.78 & $\mathbf{2 2 . 7 8}$ \\
\hline Einstein & 27.91 & 19.92 & $\mathbf{1 5 . 9 2}$ \\
\hline Couple & 21.17 & 13.23 & $\mathbf{9 . 2 2}$ \\
\hline Girl & 17.04 & 9.04 & $\mathbf{5 . 0 4}$ \\
\hline House & 17.88 & 9.97 & $\mathbf{5 . 9 1}$ \\
\hline Clock & 10.03 & 2.10 & $\mathbf{1 . 8 8}$ \\
\hline Peppers & 25.46 & 14.21 & $\mathbf{9 . 6}$ \\
\hline
\end{tabular}

From Table1 it is observed that the proposed method (HVSEDBI)preserves the local content of the input image in the output image better than the existing methods. In all the images a significant decrease in the AMBE values is observed making the proposed method better than the existing methods for local content preservation .

Table 2 Comparison of the PSNR values produced by (HVS) and HVSedge and the proposed method HVSEDBI

\begin{tabular}{|l|l|l|l|}
\hline \multirow{2}{*}{ Image } & \multicolumn{3}{|c|}{ PSNR } \\
\cline { 2 - 4 } & HVS & HVSedge & HVSEDBI \\
\hline Lena & 25.28 & 25.95 & $\mathbf{2 6 . 3 0}$ \\
\hline Einstein & 25.64 & 26.31 & $\mathbf{2 6 . 7 0}$ \\
\hline Couple & 26.40 & 26.98 & $\mathbf{2 7 . 3 0}$ \\
\hline Girl & 26.09 & 28.31 & $\mathbf{2 9 . 5 2}$ \\
\hline House & 26.45 & 27.30 & $\mathbf{2 7 . 8 8}$ \\
\hline Clock & 27.90 & 30.54 & $\mathbf{3 1 . 6 2}$ \\
\hline Peppers & 25.94 & 26.67 & $\mathbf{2 7 . 2 6}$ \\
\hline
\end{tabular}

From the above table (table2) it is observed that the the PSNR values obtained for the proposed method is better compared to the values obtained for the existing methods indicating that the proposed method generates images of better quality compared to the existing methods.

The following images (Figure 1) shows examples of outputs produced by the existing methods and the proposed method((HVSEDBI) for standard images .
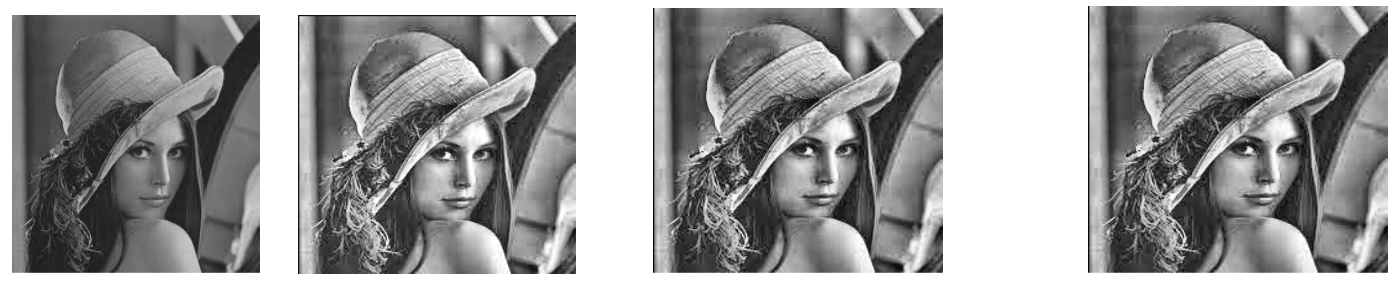

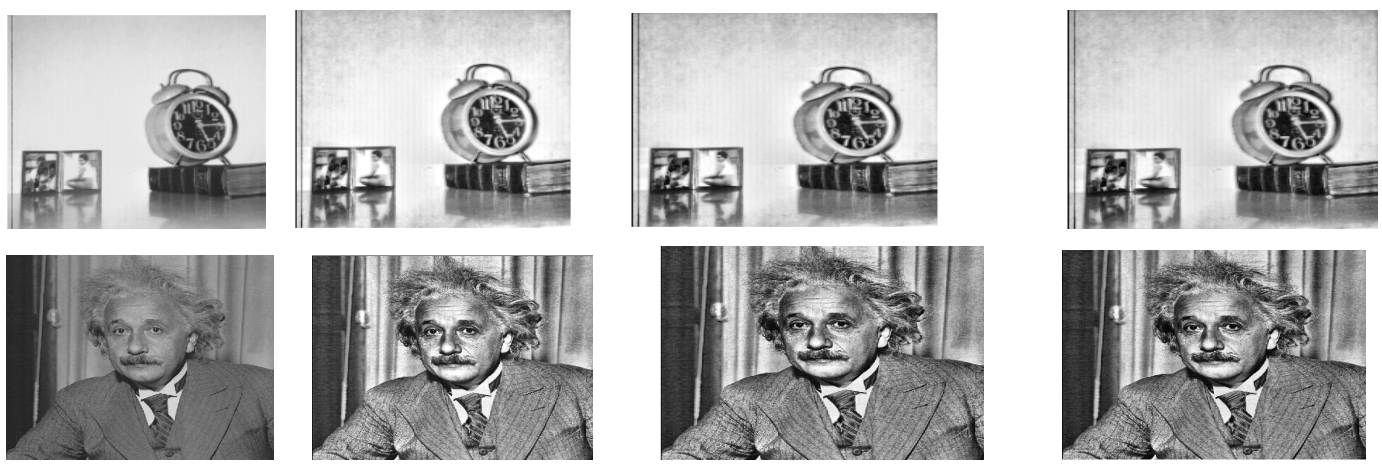

(a)

(b)

(c)

(d)

Figure.1 Column(a) Original Images Lena ,Clock ,Einstein Column (b) HVS Column (c) Output using HVSedge Column (d) Output using HVSEDBI

Since local content preservation is a major factor in the case of medical images ,the proposed method (HVSEDBI) is also applied on medical images .The table below gives the results obtained likewise .

Table 3 Comparison of the AMBE values produced by (HVS) and HVSedge and the proposed method HVSEDBI for medical images

\begin{tabular}{|l|l|l|l|}
\hline \multirow{2}{*}{ Image } & \multicolumn{3}{|c|}{ AMBE } \\
\cline { 2 - 4 } & HVS & HVSedge & HVSEDBI \\
\hline Knee1 & 21.43 & 13.47 & $\mathbf{7 . 2 7}$ \\
\hline Knee4 & 27.51 & 19.53 & $\mathbf{1 4 . 1 5}$ \\
\hline Mammo1 & 26.00 & 18.02 & $\mathbf{1 2 . 7 7}$ \\
\hline Mammo2 & 35.36 & 27.37 & $\mathbf{1 2 . 6 8}$ \\
\hline Brain1 & 18.86 & 12.86 & $\mathbf{6 . 1 8}$ \\
\hline Brain2 & 24.49 & 18.51 & $\mathbf{1 1 . 7 0}$ \\
\hline Brain3 & 23.05 & 17.05 & $\mathbf{9 . 5}$ \\
\hline Brain4 & 21.62 & 15.63 & $\mathbf{8 . 0 8}$ \\
\hline Mammo0 & 27.66 & 19.67 & $\mathbf{1 5 . 3 4}$ \\
\hline
\end{tabular}

As mentioned before , a method is said to preserve the local content of an input image in the output image better if the AMBE value generated by it for an image is low .From the above table it is seen that there is significant decrease in the AMBE value generated by the proposed method compared to the existing methods which in turn is very essential in the case of medical images.

Table 4 Comparison of the PSNR values produced by (HVS) and HVSedge and the proposed method HVSEDBI for medical images

\begin{tabular}{|l|l|l|l|}
\hline \multirow{2}{*}{ Image } & \multicolumn{3}{|c|}{ PSNR } \\
\cline { 2 - 4 } & HVS & HVSedge & HVSEDBI \\
\hline Knee1 & 25.21 & 26.21 & $\mathbf{2 8 . 2 8}$ \\
\hline Knee4 & 24.84 & 26.01 & $\mathbf{2 7 . 9 9}$ \\
\hline Mammo1 & 25.25 & 26.33 & $\mathbf{2 7 . 7 8}$ \\
\hline Mammo2 & 24.31 & 24.79 & $\mathbf{2 8 . 9 7}$ \\
\hline
\end{tabular}




\begin{tabular}{|l|l|l|l|}
\hline Brain1 & 25.76 & 27.26 & $\mathbf{3 0 . 1 8}$ \\
\hline Brain2 & 25.43 & 26.74 & $\mathbf{2 9 . 3 3}$ \\
\hline Brain3 & 25.41 & 26.76 & $\mathbf{2 9 . 6 4}$ \\
\hline Brain4 & 25.42 & 26.76 & $\mathbf{2 9 . 9 6}$ \\
\hline Mammo0 & 24.91 & 26.94 & $\mathbf{2 8 . 0 2}$ \\
\hline
\end{tabular}

From the above table it can be observed that is a significant increase in the PSNR values obtained by the proposed methods (HVSEDBI) compared to the existing methods for medical images also.

The following images (Figure 2) shows examples of outputs produced by the existing methods and the proposed method ((HVSEDBI) in the different category of medical images .
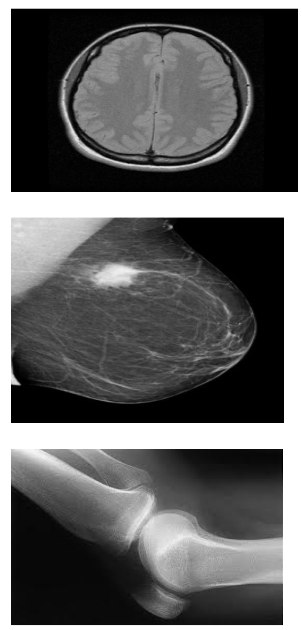

(a)
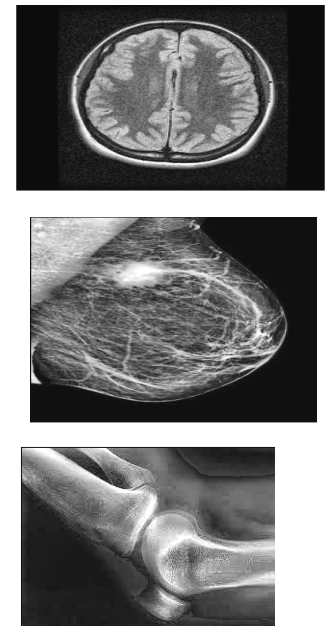

(b)
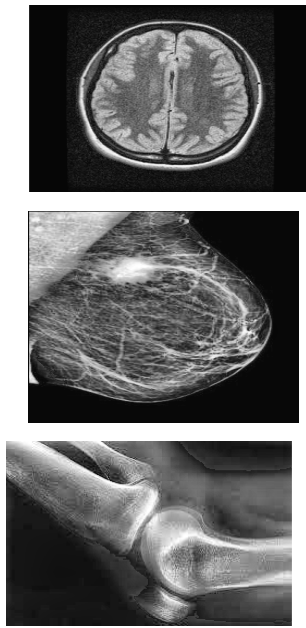

(c)
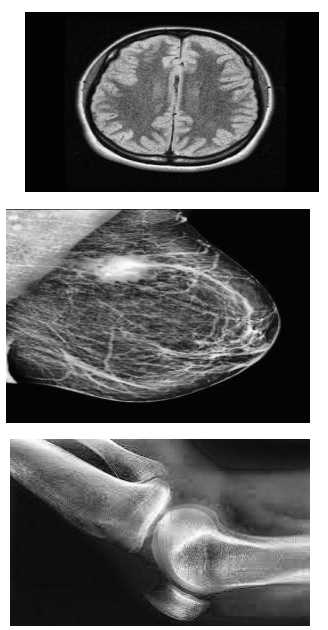

(d)

Figure.2 Column(a)Original Imagesbrain1,mammo0,knee1 Column (b) HVS Column (c) Output using HVSedge Column (d) Output using HVSEDBI

Finally since the proposed methods and the existing methods used for comparison are enhancement methods, a comparison of the enhancement in the contrast obtained by the above methods using a performance measure called contrast improvement index(CII)is shown in Table 5 .

Table 5 Comparison of the CII values produced by (HVS) and HVSedge and the proposed method HVSEDBI for standard images

\begin{tabular}{|l|l|l|l|}
\hline \multirow{2}{*}{ Image } & \multicolumn{3}{|c|}{ CII } \\
\cline { 2 - 4 } & HVS & HVSedge & HVSEDBI \\
\hline Lena & 1.51 & 1.6 & $\mathbf{1 . 7 4}$ \\
\hline Einstein & 2.2 & 2.4 & $\mathbf{2 . 5 3}$ \\
\hline Couple & 2.0 & 2.1 & $\mathbf{2 . 2}$ \\
\hline Girl & 2.2 & 2.4 & $\mathbf{2 . 5}$ \\
\hline House & 1.52 & 1.6 & $\mathbf{1 . 7 9}$ \\
\hline Clock & 1.63 & 1.76 & $\mathbf{1 . 8 4}$ \\
\hline Peppers & 1.06 & 1.51 & $\mathbf{1 . 5 6}$ \\
\hline
\end{tabular}




\section{CONCLUSION}

In this paper, a HVS based image enhancement method is introduced. A number of enhancement algorithms can be used with HVS based methods .Here an enhancement algorithm for dark blurred images is used with $\mathrm{H}$ uman Vision thresholding (HVSEDBI) mainly for the preservation of local content of the images . The performance measures used for analysis are Average mean brightness error(AMBE) and Peak signal to noise ratio (PSNR).A lower value of AMBE indicates a good preservation of local content by a method while a higher value of PSNR indicates that the image generated is of good quality. Experiments on real medical images such as brain, mammogram images and knee images shows the superiority of the proposed method (HVSEDBI)over the existing methods (HVS and HVSedge ) in local content preservation. The results obtained for standard images other than the medical images shows that the proposed method is even efficient for those images also.

\section{REFERENCES}

[1] A. Rosebfield, A. C. Kak, Digital Picture processing,Academic press, San Diego, CA, 1976.

[2] R. C. Gonzalez, Richard E. Woods, Digital Imageprocessing, Addision-Wesely, 2003

[3] R. H. Sherrier, G. A. Johnson, "Regionally adaptive histogram equalization of the chest," IEEE Trans.Med.Image, Mi-6(1987), pp.1-7.

[4] S. M. Pizer, E. P. Amburn, “Adaptive histogram equalization and its variations," Compt. Vision, Graph, Image Process,39(1987), pp. 355-368.

[5] Md. Foisal Hossain, Mohammad Reza Alsharif"Image Enhancement Based on Logarithmic Transform Coefficient and Adaptive Histogram Equalization", 2007 International Conference on Convergence Information Technology.

[6] Petrou Maria, Bosdogianni Panagiota. Image Processing: The Fundamentals. John Wiley and Sons; 1999.

[7] Zimmerman John B, Pizer Stephen M, Staab Edward V, Randolph Perry J, McCartney William, Brenton Bradley C. An evaluation of the effectiveness of adaptive histogram equalization for contrast enhancement. IEEE Trans Med Imag 1988;7(4):04-312.

[8] Kim Joung-Youn, Kim Lee-Sup, Hwang Seung-Ho. An advanced contrast enhancement using partially overlapped sub-block histogram equalization.IEEE Trans Circuits Syst Video Technol 2001;11(4):475-84.

[9] Pizer Stephen M, Philip Amburn E, Austin John D, Cromartie Robert, Geselowitz Ari, Greer Trey, et al. Adaptive histogram equalization and its variations. Comput Vision Graph Imag Process 1987;39(3):355-68.

[10] Pizer Stephen M, Johnston R. Eugene, Ericksen P. James, Bonnie C. Yankaskas, Muller Keith E. Contrast-limited adaptive histogram equalization speed and effectiveness. In: Proceedings of the .rst conference on visualization in biomedical computing, 1990. p. 337-45.

[11] Naglaa Yehya Hassan1, and Nori Aakamatsu,"Contrast Enhancement Technique of dark blurred image, IJCSNS International Journal of Computer Science and Network Security, VOL.6 No.2A, February 2006.

[12] Karen A. Panetta, Fellow, IEEE, Eric J. Wharton, Student Member, IEEE, and Sos S. Agaian, Senior Member, IEEE,"Human visual system- based image enhancement and logarithmic contrast measure", 
IEEE TRANSACTIONS ON SYSTEMS, MAN, AND CYBERNETICS-PART B: CYBERNETICS, VOL. 38, NO. 1, FEBRUARY 2008

[13] Karen A. Panetta, Fellow, IEEE, Eric J. Wharton,’Human visual system based multi histogram equalization for non uniform illumination and shadow correction",ICASSP2007

[14] Md. Foisal Hossain, Mohammad Reza Alsharif“Image Enhancement Based on Logarithmic Transform Coefficient and Adaptive Histogram Equalization”, 2007 International Conference on Convergence Information Technology

[15] Kim Joung-Youn, Kim Lee-Sup, Hwang Seung-Ho,” An advanced contrast enhancement using partially overlapped sub-block histogram equalization",IEEE Trans Circuits Syst Video Technol 2001;11(4):475-84.

[16] S. M. Pizer, E. P. Amburn, "Adaptive histogram equalization and its variations," Compt. Vision, Graph, Image Process,39(1987), pp. 355-368.

[17] Petrou Maria, Bosdogianni Panagiota. Image Processing: The Fundamentals. John Wiley and Sons; 1999.

[18] Zimmerman John B, Pizer Stephen M, Stab Edward V, Randolph Perry J, McCartney William, Brenton Bradley C, "An evaluation of the effectiveness of adaptive histogram equalization for contrast enhancement” IEEE Trans Med Imag 1988;7(4):04-312.

[19] Pizer Stephen M, Philip Amburn E, Austin John D, Cromartie Robert, Geselowitz Ari, Greer Trey, et al. Adaptive histogram equalization and its variations. Comput Vision Graph Imag Process 1987;39(3):355-68.

[20] Y.-T. Kim, "Contrast enhancement using brightness preserving bi histogram equalization," IEEE Trans. on Consumer Electronics, vol. 43,no. 1, pp. 1-8, Feb. 1997.

[21] Y. Wang, Q. Chen, and B. Zhang, "Image enhancement based on equal area dualistic sub-image histogram equalization method," IEEE Trans. on Consumer Electronics, vol. 45, no. 1, pp. 68-75, Feb. 1999.

[22] S.D. Chen and A. Ramli, "Minimum mean brightness error bi-histogram equalization in contrast enhancement," IEEE Trans. on Consumer Electronics, vol. 49, no. 4, pp. 1310-1319, Nov. 2003.

[23] S.-D. Chen and A. Ramli, "Contrast enhancement using recursive mean separate histogram equalization for scalable brightness preservation,"IEEE Trans. on Consumer Electronics, vol. 49, no. 4, pp. 1301-1309,Nov. 2003. 